\title{
Study on Dynamic Multi-objective Emergency Decision -making Model of Social Emergency
}

\author{
Hongyan $\mathrm{Li}^{1, \mathrm{a}}$, Daoping $\mathrm{Zhu}^{* 2, \mathrm{~b}}$ \\ ${ }^{1}$ School of Management Guangdong University of Technology Guangzhou, China \\ ${ }^{2}$ School of Management Guangdong University of Technology Guangzhou, China
}

\begin{abstract}
According to the characteristics of emergencies, a dynamic multi-objective emergency decisionmaking model is proposed. By introducing triangular fuzzy Numbers to calculate the correlation distance between the selected solution, the ideal solution and the negative ideal solution, considering the correlation degree between solutions, the correlation between indexes is highlighted. In addition, the paper strengthens the processing of fuzzy and uncertain information in the real world through the comprehensive integrated fuzzy theory, which is closer to the practical application. Finally, the paper combines the example of emergency decision making of African swine fever accident, and found that the method is relatively scientific through the simulation calculation by the expert group from the invited school.
\end{abstract}

\section{Introduction}

In recent years, the natural environment has become extremely unbalanced due to the accelerated economic development, and the stability of the environment has been damaged.

There are more and more natural disasters around the world every year, and each natural disaster brings huge losses to the country and society, So far the number of confirmed cases of COVID-19 has exceeded one million globally, and people all over the world are deeply affected by COVID-19. In fact, novel Coronavirus is just one of the global pandemics we have been experiencing since 2018 . For example, the African swine fever outbreak in China since August 2018, the Bushfire outbreak in Australia in June 2019, the dengue fever outbreak in South America since February 2020, the influenza B virus outbreak in the United States, high-risk avian influenza outbreaks in many countries, and locust plague in Africa. It is more and more important to respond to emergencies quickly, take emergency actions in time, and eliminate the destructive potential of emergencies.

Emergency decision-making has become a part of the political management of western capitalist society [1]. China also issued the National General Emergency Plan for Public Emergencies and the Emergency Response Law of the People's Republic of China from 2006 to 2007, which promoted the decision-making for emergencies to the level of unified national management.

\section{Literature review}

Due to the shortage of information and sudden occurrence of emergencies, and the complex and changeable environment, many decision-making methods and models are put forward at present. Based on the classical decision theory, scholars at home and abroad have studied the optimization model theory in emergency decision making, and paid more attention to the quantitative analysis methods such as system theory, game theory, operations research, mathematical statistics, model and simulation, which have provided the possibility for scientific decision making. On the basis of considering the psychology of decision makers, L proposed a risk decision analysis method of TODIM method [2]. Rahman et al. used the organizational decision model and method to form a coherent matrix, and discussed the emergency decision model and method in the emergency situation [3]. Yu proposed a distance-based group decision making method for unconventional multi-person and multi-criterion emergency decision making [4]. Liu et al. proposed a risk multi-attribute decision making method based on multiple states and different results of emergency evolution [5]. Amailef proposed an ontology method to support the emergency decision system based on case reasoning in view of the deficiency of the existing emergency decision support system, and conducted experiments to support the emergency decision makers to effectively respond to emergencies [6]. Xu et al. proposed a consensus model for large group emergency decision to deal with large-scale emergencies [7]. Mendonca et al. (2000) believed that an effective emergency response has two key factors, namely, the implementation speed of response strategy and the knowledge quality possessed by experts, so they proposed a knowledge method using computer technology and communication technology to improve the emergency response capability [8].

Although scholars studied the emergency decision summary by using the method of the innovation for the future research development direction, promote and

a-mail: olina@gdut.edu.cn

b* Correspondence author: 1367786804@qq.com 
practice played an irreplaceable role, however, the above detailed combing literature, we found that there is still a lack of the following: (1) Consider less literature of emergency decision face a number of possible states; (2) At the disciplinary level, most of the existing research results are focused on computer software and application and engineering technology, but few are conducted by using modern decision-making methods. (3) Although emergency decision-making research is rich, but in natural disasters, accidents disasters, public health emergencies such as frequent Chinese localization of application, regardless of the influencing factors, consequences and emergency mechanism to the west is very different, investigate its reason is China's specific national conditions, inadequate emergency environment and lack of effective media, therefore, we need to put forward a suitable solution for China's national conditions of emergency decision-making plan. (4) The current research methods focus on a single method. For example, Fu studied the TOPSIS based emergency decision optimization problem and verifies it through the case of flood control dispatching emergency decision [9]. Tian Jun et al. (2011) determined the demand for emergency supplies by means of triangular fuzzy Numbers, and established a targeted multi-objective mathematical model for dynamic dispatching of emergency supplies distribution [10].

Above various methods for the solution of the problem has made irreplaceable role, but in view of the current complex problem in China, a single method is used to solve the problem of emergency there may have limitations, lack of universality, in this paper, in order to make more scientific research methods, and provide a comprehensive solution to more emergency accidents, so we studied three methods such as Markov chain prediction, triangular fuzzy number, TOPSIS evaluation. Based on this, this paper proposed a multi-objective emergency decision-making method for emergency response facing multiple possible circumstance.
The paper is organized as follows: In Section 2, we describe the methodology. In Section 3, African swine fever (ASF) was taken as an example to verify the method. In Section 4, we finally present the discussion and conclusions.

\section{Theoretical basis}

\subsection{Markov prediction method}

Emergency is usually a multi-state risk decision problem, each state is random and has Markov property, so it is suitable to use Markov prediction method for analysis. Markov prediction method does not need a large number of statistical data, only a part of recent data can be used to achieve the prediction, greatly reducing the difficulty of information collection, has been widely used in computational mathematics, finance and economics, humanities and other fields.

The concept of Markov chain was first proposed by The Russian mathematician Markov in the early 20th century. Markov chain is a random process with markov property which is discrete in time and discrete in state. Given its present state (present), its future evolution (future) does not depend on its past evolution (past), i.e., [11]. This method has two important concepts: (1) Random motion system: at any moment, the state of the system is random, then the change process of the system is a random process; (2) State transition probability: Since the transition of each state of the system is random, it is necessary to use probability to describe the probability that occurs when the system is randomly transferred from one state to another.

The following table 1 is a brief description of how Markov prediction method predicts the probability of accidents occurring in the next phase of the system:

1) Variable definition:

Table1. Variable definition table

\begin{tabular}{c|c|c}
\hline Variables represent & meaning & constraints \\
\hline $\mathrm{t}$ & current state & No \\
\hline $\mathrm{t}+1$ & next state & No \\
\hline $\mathrm{Pij}$ & Probability of Pij random accident converting into j state at time $\mathrm{t}$ in I state & {$[0,1]$} \\
\hline
\end{tabular}

2) Conditional probability:

$$
\mathrm{Pij}=\mathrm{P}\{\mathrm{Xt}+1=\mathrm{it}+1 \mid \mathrm{Xt}=\mathrm{it}\}
$$

3) Suppose the initial state vector of the system is $\mathrm{P}(1)=($ pi1,pi1,..,pn1), then the next state vector of the system is:

$$
P(t+1)=P(t) \times P
$$

Where $\mathrm{P}(\mathrm{t})$ is the vector of the previous state, and $\mathrm{P}$ is the probability that the accident occurs to stabilize after several steps.

\subsection{Triangular fuzzy number comprehensive evaluation method}

Fuzzy theory mainly includes fuzzy set theory, fuzzy logic, fuzzy reasoning and fuzzy control. In the traditional fuzzy comprehensive evaluation method, the membership degree of each evaluation index is unique. And comprehensive evaluation method of triangular fuzzy number, dimension of each index is not fixed index, but to make more information such as it's own experience, 
knowledge, experts in the interval $[0,1]$, the membership degree of the evaluation index is presented of the maximum and minimum, most likely values, instead of traditional fuzzy comprehensive evaluation method of fixed evaluation scale, each evaluation index in [minimum and maximum] this fuzzy evaluation range, the area reflects the self-confident degree of expert evaluation results, the smaller area the larger the range self-confident degree.

Definition and operation rules of triangular fuzzy Numbers

Definition: $a=(a 1, a 2, a 3)$ is the triangular fuzzy number, and the membership function can be expressed as [12].

$$
\mathrm{f}_{\mathrm{a}}=\left\{\begin{array}{cc}
0 & \mathrm{x} \leq \mathrm{a}_{1} \\
\frac{\mathrm{x}-\mathrm{a}_{1}}{\mathrm{a}_{2}-\mathrm{a}_{1}} & \mathrm{a}_{1}<x \leq \mathrm{a}_{2} \\
\frac{\mathrm{x}-\mathrm{a}_{3}}{\mathrm{a}_{2}-\mathrm{a}_{3}} & \mathrm{a}_{1}<x \leq \mathrm{a}_{2} \\
0 & \mathrm{x}>\mathrm{a}_{3}
\end{array}\right.
$$

Where $x \in R, a_{1}<a_{2}, a_{3}, a_{1}$ and $a_{3}$ are upper bound and lower bound, $a_{1}$ and $a_{3}$ represent the degree of ambiguity, $a_{3}-a_{1}$ is bigger, the ambiguity degree is more stronger.

Operation rule: if $f_{a 1}, f_{a 2}$ represents the membership function of two triangular fuzzy Numbers $a_{1}, a_{2}$, respectively, the membership function of the triangular fuzzy number $\mathrm{T}=\mathrm{f}\left(\mathrm{a}_{1}, \mathrm{a}_{2}\right)$, then the membership function is given by the following equation

$$
f_{T}(s)=\sup _{(x, y) \in R^{2}, z=f(x, y)}\left(\min \left(f_{a 1}(x), f_{a 1}(y)\right)\right)
$$

Based on the above formula, the relevant algorithms of triangular fuzzy numbers are as follows:

Make $M_{1}=\left(l_{1}, m_{1}, u_{1}\right), M_{2}=\left(l_{2}, m_{2}, u_{2}\right)$ are two triangular fuzzy Numbers, then:

$M_{1} \oplus M_{2}=\left(l_{1}, m_{1}, u_{1}\right) \oplus\left(l_{2}, m_{2}, u_{2}\right)=\left(l_{1}+l_{2}, m_{1}+m_{2}, u_{1}+u_{2}\right)$ $M_{1} \odot M_{2}=\left(l_{1}, m_{1}, u_{1}\right) \odot\left(l_{2}, m_{2}, u_{2}\right)=\left(l_{1} l_{2}, m_{1} m_{2}, u_{1} u_{2}\right)$ $M_{1}^{-1}=\left(l_{1}, m_{1}, u_{1}\right)^{-1}=\left(\frac{1}{l_{1}}, \frac{1}{m_{1}}, \frac{1}{u_{1}}\right)$

\subsection{TOPSIS Evaluation method}

TOPSIS evaluation method is a method to rank the proximity of a limited number of evaluation objects to the idealized goal and to evaluate the relative merits of existing objects. Its purpose is to find a scheme approaching the ideal from multiple evaluation objects by combining multiple considerations[13].

The process of applying TOPSIS evaluation method to obtain the optimal scheme. For each indicator of evaluation, this paper assumes that there are five grades, namely unimportant, generally important, relatively important, important and very important. At the same time, triangular fuzzy Numbers $(\mathrm{a}=(\mathrm{a} 1, \mathrm{a} 2, \mathrm{a} 3))$ are introduced to quantify the five grades. Here, the arithmetic value of the interval between $[0,1]$ is used for the evaluation of the five grades [14]. Finally, TOPSIS evaluation method is used to determine the [optimal, worst] combination of each index, design the positive ideal solution and negative ideal solution of each index, and then calculate the distance between each evaluation object and the positive ideal solution and negative ideal solution to select the optimal evaluation object, namely the optimal solution.

\subsubsection{Calculation steps}

The first step is to calculate the probability of the accident $\mathrm{P}=\operatorname{Pij}\left(\begin{array}{ccc}\mathrm{p} 11 & \cdots & \mathrm{p} 1 \mathrm{l} \\ \vdots & \ddots & \vdots \\ \mathrm{pt} 1 & \cdots & \mathrm{ptl}\end{array}\right)$ (Where, $\left.\mathrm{i} \in[1, \mathrm{t}], \mathrm{j} \in[1,1]\right)$

Where, Pij represents the probability that the system will be converted to state $\mathrm{L}$ within a time period $\mathrm{T}$.

The second step is to standardize the evaluation index in evaluation system with multiple indexes, each evaluation index exists to different units, dimensions, and the phenomenon of different scales, in order to avoid bring interference to the comprehensive evaluation, we need to preprocess the original evaluation, means standardized processing all evaluation indexes, let them be dimensionless, countless norm, and eliminate the deviation between the indicators, and then evaluate them. All evaluation indicators can be divided into two categories from the economic perspective, one is the efficiency indicator, the larger the better, and the other is the cost indicator, the smaller the better [15]. Here, the standardized treatment of quantitative indicators adopts linear proportional transformation, and the transformation formula is as follows:

$$
\begin{aligned}
& F \max =\max (\text { aij }) \\
& F \min =\min (\text { aij })
\end{aligned}
$$

For efficiency indicator, it is defined as follows:

$$
\mathrm{Rij}=\frac{\mathrm{aij}}{\mathrm{Fmax}}
$$

For cost indicator, it is defined as follows:

$$
\mathrm{Rij}=\frac{\text { Fmin }}{\mathrm{aij}}
$$

After this standardized treatment, the evaluation index value of each pretreatment $0 \leq \mathrm{Rij} \leq 1$ was obtained. It is easy to calculate.

The third step is to calculate the standardized decision matrix

$$
Y i j=\frac{\text { aij }}{\sum_{\mathrm{j}=1}^{\mathrm{n}} \text { aij }}(\mathrm{i} \in[1, \mathrm{~m}], \mathrm{j} \in[1, \mathrm{n}])
$$

The fourth step, to determine the weight value of each index $\mathrm{N}$, then each expert use triangular fuzzy number to score of each evaluation index, then each evaluation objects can have $\mathrm{N}$ fuzzy weighted win, to get the weighting sum calculated average as the final fuzzy weighted value of each index $\mathrm{Wj}=(\mathrm{aj}, \mathrm{bj}, \mathrm{cj})(\mathrm{Wj}$ is in $\mathrm{J}$ status, the index's weight value), the computation formula is as follows:

$$
\begin{array}{r}
\text { aj }=\frac{1}{n} \sum_{i=1}^{n} \operatorname{win}(i \in[1, n]) \\
\text { bj }=\frac{1}{n} \sum_{i=1}^{n} \operatorname{win}(i \in[1, n]) \\
\text { cj }=\frac{1}{n} \sum_{i=1}^{n} \operatorname{win}(i \in[1, n])
\end{array}
$$

The fifth step is to standardize the fuzzy weight.

$$
w j=\frac{a j}{\sum_{j=1}^{n} \text { aj }}(a j=\sqrt[3]{a j * b j * c j}(j \in[1, n]))
$$

The sixth step is to calculate the weighted decision matrix.

$$
\mathrm{O}=\left(\begin{array}{ccc}
011 & \cdots & \mathrm{o} 1 \mathrm{n} \\
\vdots & \ddots & \vdots \\
\text { om1 } & \cdots & \text { omn }
\end{array}\right)(\mathrm{oij}=\mathrm{Yij} * w \mathrm{w}, \mathrm{i} \in[1, \mathrm{~m}], \mathrm{j} \in[1, \mathrm{n}])
$$


The seventh step is to calculate the positive ideal solution $\mathrm{O}^{+}$and negative ideal solution $\mathrm{O}^{-}$for each solution.

According to the obtained weighted decision matrix, the positive ideal solution is the maximum value of each column in the matrix. The negative ideal solution is the minimum value of each column in the matrix. By taking the value of each column of the weighted decision matrix, the positive understanding matrix and the negative ideal solution matrix of each evaluation object can be obtained.

$$
\begin{aligned}
& \mathrm{O}^{+}=\left(\begin{array}{ccc}
\mathrm{o} 11^{+} & \cdots & \mathrm{o} 1 \mathrm{n}^{+} \\
\vdots & \ddots & \vdots \\
\mathrm{om} 1^{+} & \cdots & \mathrm{omm}^{+}
\end{array}\right) \quad\left(0^{+}=\left\{\mathrm{o} 1^{+}, \mathrm{o} 2^{+}, \cdots, \mathrm{on}^{+}\right\}=\right. \\
& \left.\left\{\left(\max _{\mathrm{j}} \mathrm{oij}, \mathrm{j} \in \in^{\mathrm{n}}\right)\right\}\right)(14) \\
& \mathrm{O}^{+}=\left(\begin{array}{ccc}
\mathrm{o} 11^{+} & \cdots & \mathrm{o} 1 \mathrm{n}^{+} \\
\vdots & \ddots & \vdots \\
\mathrm{om} 1^{+} & \cdots & \mathrm{omm}^{+}
\end{array}\right) \quad\left(0^{-}=\left\{\mathrm{o} 1^{-}, \mathrm{o} 2^{-}, \cdots, \mathrm{on}^{-}\right\}=\right. \\
& \left.\left\{\left(\min _{\mathrm{j}} \mathrm{oij}, \mathrm{j} \in \in^{\mathrm{n}}\right)\right\}\right)(
\end{aligned}
$$

\section{Example analysis}

Based on case analysis African swine fever outbreak in China in 2018, in the case as the research object for two reasons: reason one is that China's pig power, pork consumption power, live pig market volume, breeding stock are the biggest in the world, and pork consumption and imports of breeding pigs are tremendous every year, and trade with many countries are frequent, Since 2020, a total of 2,346 pigs and 7,556 wild boar outbreaks have occurred in 27 countries and regions (new Laos), and a total of 9,902 cases of ASFV have been reported. However, until the beginning of 2020, there is still no specific vaccine or antiviral drug for ASFV that can effectively control the spread of the virus in a timely manner during the outbreak, it had brought huge risks to the country and the whole world, and the direct and indirect losses will be incalculable. Second, ASF was introduced into China in August 2018. The disaster brought by the virus epidemic tends to bring panic to the people, which will prompt people to take unpredictable actions, which in turn will bring a series of unpredictable accidents to the country and society. Therefore, a good decision on emergency handling to the outbreak of African swine fever can not only reduce the occurrence of unpredictable accidents for the country and society, but also provide reference and learning for other countries, so as to minimize the risk of epidemic infection.

\subsection{A case study of the outbreak of African swine fever}

The spread of the virus is uncertain, and the impact of epidemic development on people is hard to predict. We can only predict that the spread of epidemic in the next stage and the impact on people's behavior based on current situation of the spread of epidemic. Therefore, we can use Markov prediction method to predict occurrence probability of future accidents.

According to Markov's prediction method, we can take China as a system to predict the occurrence probability of African swine fever (AFCD) emergencies in each stage of development.

After the discovery of AFCD, the Ministry of Agriculture and Rural Affairs of The People's Republic of China classified the epidemic situation in Africa into four levels according to epidemic characteristics, the harm degree and the scope of the epidemic situation were named as a particularly major accident (1), a major accident (2), a relatively major accident (3) and a general accident (4). In order to make the whole research method more robust, we invited part-time MBA students from emergency department and leaders of some related fields to form a simulated expert group. Expert group scored four levels of transfer probability. The results are shown in the table 2 below.

Table2. Assumes the probability of state transition of expert group to an AFCD

\begin{tabular}{c|c|c|c|c}
\hline Accident level & Special major accident & $\begin{array}{c}\text { Major accident } \\
\text { Relatively }\end{array}$ & Major accident & General accident \\
\hline Special major accident & 0.2 & 0.2 & 0.4 & 0.3 \\
\hline Major accident Relatively & 0.2 & 0.3 & 0.2 & 0.2 \\
\hline Major accident & 0.1 & 0.1 & 0.1 & 0.2 \\
\hline General accident & 0.1 & 0.1 & 0.3 \\
\hline
\end{tabular}

If change to probability matrix,

$$
\mathrm{P}=\left[\begin{array}{llll}
0.2 & 0.2 & 0.4 & 0.3 \\
0.2 & 0.3 & 0.2 & 0.2 \\
0.1 & 0.1 & 0.2 & 0.2 \\
0.1 & 0.1 & 0.1 & 0.3
\end{array}\right]
$$

Based on the experts' input scores, we can know that the probability of a significant accident to become a relatively significant accident is 0.2 , and the probability of a normal accident to become a relatively significant accident is 0.1 .

According to the evolution characteristics of emergency, based on the classic theory of life cycle put forward by Steven Fink, a famous American crisis management expert, the simulation expert group unanimously believe that the development of AFCD can be divided into four stages: The incubation period, The outbreak period, The extension period and the recovery period. The characteristics of African swine fever in each stage: the incubation period is characterized by point-like occurrence and long-distance leaping transmission; In the outbreak period, the regional African swine fever was spread from point to surface. The extension period shows multi-point distribution; The recovery period showed that there was no African swine fever epidemic everywhere.

After team discussion and brainstorming, they think that the initial state is usually low grade, therefore incubation period calculated by the matrix, we can put the 
initial state of probability value is set to $\mathrm{P} 1=(0,0,0,1)$, according to the formula of Markov prediction method (1), we can respectively calculate probability of each stage of development, the second phase state probability $\mathrm{P} 2=\mathrm{P} *$ $\mathrm{P}=(0.1,0.1,0.1,0.3)$, the third stage state probability $\mathrm{P} 3$ $=\mathrm{P} 2 * \mathrm{P}=(0.06,0.09,0.11,0.16)$, the fourth stage state probability of $\mathrm{P} 4=\mathrm{P} 3 * \mathrm{P}=(0.057,0.066,0.080,0.106)$. Finally, the probability matrix $\mathrm{Z}$ can be obtained in four stages of incubation period, outbreak period, extension period and recovery period:

$$
\mathrm{Z}=\left[\begin{array}{clll}
0 & 0 & 0 & 1 \\
0.1 & 0.1 & 0.1 & 0.3 \\
0.06 & 0.09 & 0.11 & 0.16 \\
0.057 & 0.066 & 0.080 & 0.106
\end{array}\right]
$$

After the above probabilities were obtained, we invited experts again to propose their own plans based on the consideration of five factors including time, cost, feasible probability, risk index and effect by using brainstorming method and combining with the actual situation. After discussion, 4 groups of emergency plans are selected as shown in Table 3 below.

Table3. Indicator evaluation of four emergency solutions

\begin{tabular}{c|c|c|c|c|c}
\hline Solutions & Time (hours) & Cost $(10,000 \mathrm{rmb} /$ yuan $)$ & Feasible possibility & Risk index (10 point scale) & Effect (100 point scale) \\
\hline 1 & 56 & 450 & 0.58 & 7 & 78 \\
\hline 2 & 78 & 590 & 0.79 & 6 & 87 \\
\hline 3 & 80 & 380 & 0.82 & 3 & 80 \\
\hline 4 & 68 & 520 & 0.77 & 9 & \\
\hline
\end{tabular}

If solution 1 is adopted in the African swine fever accident, time required to control whole accident is 56 hours and cost is 4.5 million yuan. The feasibility of the plan is $58 \%$, and high risk index reached 7 . Overall effect is relatively good.

According to the standardized treatment formulas (4) $\sim(7)$, data in Table 2 are standardized which time, cost and risk index belong to cost-type index, feasible probability and effect belong to efficiency-type index. After eliminating dimensional differences, normalized matrix is obtained as table 4 below.

According to Table 4, standardized decision matrix is calculated according to Formula (8). Calculation results are shown in following table5:

Table4. Indicator evaluation of four emergency solutions after dimension differences elimination

\begin{tabular}{c|c|c|c|c|c}
\hline Solutions & $\begin{array}{c}\text { Time } \\
\text { (hours })\end{array}$ & Cost $(10,000 \mathrm{rmb} / \mathrm{yuan})$ & Feasible possibility & $\begin{array}{c}\text { Risk index (10 point scale ) } \\
\text { scale })\end{array}$ \\
\hline 1 & 1 & 0.844444444 & 0.707317073 & 0.428571429 \\
\hline 2 & 0.717948718 & 0.644067797 & 0.963414634 & 0.5 & 0.666666667 \\
\hline 3 & 0.7 & 1 & 0.96666667 & 0.888888889 & 1 \\
\hline 4 & 0.823529412 & 0.730769231 & 0.93902439 & 1 \\
\hline
\end{tabular}

Table5. Indicators evaluation of four standardized emergency response solutions

\begin{tabular}{c|c|c|c|c|c}
\hline Solutions & Time (hours) & Cost $(10,000 \mathrm{rmb} / \mathrm{yuan})$ & Feasible possibility & Risk index (10 point scale) & $\begin{array}{c}\text { Effect }(100 \text { point } \\
\text { scale })\end{array}$ \\
\hline 1 & 0.061269594 & 0.051738768 & 0.043337 & 0.026258397 & 0.053100315 \\
\hline 2 & 0.043988427 & 0.039461773 & 0.059028 & 0.030634797 & 0.059227274 \\
\hline 3 & 0.042888716 & 0.061269594 & 0.06127 & 0.036761756 & 0.061269594 \\
\hline 4 & 0.050457313 & 0.044773934 & 0.057534 & 0.061269594 & \\
\hline
\end{tabular}

According to obtained standardized index evaluation, the simulation expert group carried out triangular fuzzy number evaluation for each index of each level, and substituted the values into formulas $(9) \sim(13)$ for calculation. Calculation results are shown in following tables (table 6 to 10 ):

Table6. Evaluation of triangular fuzzy numbers of modeling expert group

\begin{tabular}{c|c|c|c|c|c}
\hline Level & Time (hours) & Cost $(10,000 \mathrm{rmb} /$ yuan $)$ & Feasible possibility & Risk index (10 point scale ) & Effect (100 point scale ) \\
\hline 1 & $0.3,0.5,0.7$ & $0.0,0.1,0.3$ & $0.1,0.3,0.5$ & $0.7,0.9,1.0$ & $0.5,0.7,0.9$ \\
\hline 2 & $0.1,0.3,0.5$ & $0.3,0.5,0.7$ & $0.0,0.1,0.3$ & $0.7,0.9,1.0$ & $0.5,0.7,0.9$ \\
\hline 3 & $0.3,0.5,0.7$ & $0.5,0.7,0.9$ & $0.7,0.9,1.0$ & $0.0,0.1,0.3$ & $0.1,0.3,0.5$ \\
\hline 4 & $0.0,0.1,0.3$ & $0.7,0.9,1.0$ & $0.5,0.7,0.9$ & $0.1,0.3,0.5$ & $0.3,0.5,0.7$ \\
\hline
\end{tabular}

Table7. Calculation results of weighted decision matrix of grade 1

\begin{tabular}{c|c|c|c|c}
\hline 0.001808 & 0.00977 & 0.001695 & 0.007632 & 0.006935 \\
\hline 0.008643 & 0.008565 & 0.007649 & 0.00816 & 0.004799 \\
\hline 0.001103 & 0.004674 & 0.008787 & 0.005075 & 0.009858 \\
\hline 0.008582 & 0.005465 & 0.008164 & 0.004493 & 0.00776 \\
\hline
\end{tabular}

Table8. Calculation results of weighted decision matrix of grade 2

\begin{tabular}{c|c|c|c|c}
\hline 0.00065 & 0.002383 & 0.009776 & 0.004175 & 0.008815 \\
\hline 0.002372 & 0.000575 & 0.000856 & 0.008511 & 0.0091 \\
\hline
\end{tabular}




\begin{tabular}{c|c|c|c|c}
\hline 0.009073 & 0.007934 & 0.007011 & 0.005094 & 0.006707 \\
\hline 0.00298 & 0.006044 & 0.009267 & 0.006778 & 0.001664 \\
\hline
\end{tabular}

Table9. Calculation results of weighted decision matrix of grade 3

\begin{tabular}{c|c|c|c|c}
\hline 0.004869 & 0.002459 & 0.008402 & 0.007221 & 0.004144 \\
\hline 0.005604 & 0.002011 & 0.006575 & 0.001611 & 0.002362 \\
\hline 0.002871 & 0.004542 & 0.003989 & 0.003919 & 0.0038 \\
\hline 0.008591 & 0.009128 & 0.002621 & 0.008652 & 0.001785 \\
\hline
\end{tabular}

Table10. Calculation results of weighted decision matrix of grade 4

\begin{tabular}{c|c|c|c|c}
\hline 0.001812 & 0.00874 & 0.008748 & 0.000425 & 0.009243 \\
\hline 0.009983 & 0.005158 & 0.007427 & 0.004742 & 0.003644 \\
\hline 0.007726 & 0.003656 & 0.000566 & 0.005037 & 0.006517 \\
\hline 0.00444 & 0.000362 & 0.00919 & 0.00441 & 0.004248 \\
\hline
\end{tabular}

Finally, according to formula (14) and (15), positive ideal solution and negative ideal solution of decision solution proposed by each expert group at four levels are calculated, and solution results are shown in following table 11 and 12:

Table11. Positive ideal solutions of decision scheme under four levels of state

\begin{tabular}{c|c|c|c|c|c}
\hline Level & & & & & \\
\hline 1 & 0.008643 & 0.00977 & 0.008787 & 0.00816 & 0.009858 \\
\hline 2 & 0.009073 & 0.007934 & 0.009776 & 0.008511 & 0.0091 \\
\hline 3 & 0.008591 & 0.009128 & 0.008402 & 0.008652 & 0.004144 \\
\hline 4 & 0.009983 & 0.00874 & 0.00919 & 0.005037 & 0.009243 \\
\hline
\end{tabular}

Table12. Negative ideal solution of decision scheme under four levels of state

\begin{tabular}{c|c|c|c|c|c}
\hline Level & & & & & \\
\hline 1 & 0.001103 & 0.004674 & 0.001695 & 0.004493 & 0.004799 \\
\hline 2 & 0.00065 & 0.000575 & 0.000856 & 0.004175 & 0.001664 \\
\hline 3 & 0.002871 & 0.002011 & 0.002621 & 0.001611 & 0.001785 \\
\hline 4 & 0.001812 & 0.000362 & 0.000566 & 0.000425 & 0.003644 \\
\hline
\end{tabular}

\subsection{Decision making recommendation}

According to the positive and negative ideal solutions of four levels, we can calculate the difference of four solutions put forward by experts group. Firstly, the [optimal and worst] combination of each indicator at the four levels is obtained, and solution results are shown in following table 13:

Table13. The [optimal, worst] combination of each indicator under four levels of state

\begin{tabular}{|c|c|c|c|c|c|}
\hline $\begin{array}{c}\text { Leve } \\
1\end{array}$ & Time (hours) & $\begin{array}{l}\text { Cost }(10,000 \\
\text { rmb/yuan })\end{array}$ & Feasible possibility & $\begin{array}{c}\text { Risk index (10 point } \\
\text { scale) }\end{array}$ & $\begin{array}{l}\text { Effect (100 point } \\
\text { scale) }\end{array}$ \\
\hline 1 & $\begin{array}{l}{[0.008643,} \\
0.001103] \\
\end{array}$ & {$[0.00977,0.004674]$} & $\begin{array}{c}0.008787, \\
0.001695]\end{array}$ & {$[0.00816,0.004493]$} & {$[0.009858,0.004799]$} \\
\hline 2 & {$[0.009073,0.00065]$} & {$[0.007934,0.000575]$} & $\begin{array}{l}{[0.009776,} \\
0.000856]\end{array}$ & {$[0.008511,0.004175]$} & {$[0.0091,0.001664]$} \\
\hline 3 & $\begin{array}{c}0.008591, \\
0.002871]\end{array}$ & {$[0.009128,0.002011]$} & 0.008402 & {$[0.008652,0.002621]$} & {$[0.004144,0.001785]$} \\
\hline 4 & $\begin{array}{c}0.009983, \\
0.001812]\end{array}$ & {$[0.00874,0.000362]$} & {$[0.00919,0.000566]$} & {$[0.005037,0.000425]$} & {$[0.009243,0.003644]$} \\
\hline
\end{tabular}

Finally, by solving the distance between each solution and positive ideal solution \& negative ideal solution, calculation results are shown in following table 14 and table 15:

Table14. Distance between four schemes and positive ideal solution

\begin{tabular}{c|c|c|c|c|c}
\hline Solutions & Time (hours) & Cost $(10,000 \mathrm{rmb} /$ yuan $)$ & Feasible possibility & Risk index (10 point scale) & Effect (100 point scale) \\
\hline 1 & 0.052627 & 0.041969 & 0.03455 & 0.018098 & 0.043242 \\
\hline 2 & 0.034915 & 0.031528 & 0.049252 & 0.022124 & 0.050127 \\
\hline 3 & 0.034298 & 0.052142 & 0.052868 & 0.02811 & 0.050318 \\
\hline 4 & 0.040474 & 0.036034 & 0.048344 & 0.056233 & 0.052027 \\
\hline
\end{tabular}

Table15. Distance between four schemes and negative ideal solution

\begin{tabular}{c|c|c|c|c|c}
\hline Solutions & Time (hours) & Cost $(10,000 \mathrm{rmb} /$ yuan $)$ & Feasible possibility & Risk index (10 point scale) & Effect (100 point scale) \\
\hline 1 & 0.060167 & 0.047065 & 0.041642 & 0.021765 & 0.048301
\end{tabular}




\begin{tabular}{l|l|l|l|l|l}
\hline 2 & 0.043338 & 0.038887 & 0.058172 & 0.02646 & 0.057563 \\
\hline 3 & 0.040018 & 0.059259 & 0.058649 & 0.035151 & 0.052677 \\
\hline 4 & 0.048645 & 0.044412 & 0.056968 & 0.060845 & 0.057626 \\
\hline
\end{tabular}

To sum up, it can be seen from Table 14 and Table 15 that, when the distance between solutions and the positive ideal solution is minimum, the distance between solutions and negative ideal solution is maximum, the solution is the optimal solution. Therefore, result calculated by the model is that we suggest use plan 2 as the emergency decisionmaking solution.

\section{Conclusions and Conclusions}

It is difficult for us to predict the occurrence of emergencies, but what kind of emergency handling decisions we should take after emergencies happen can be aided by scientific decision-making models, so as to minimize the losses brought to the country and society.

Effective emergency decision-making method can provides decision-making support for the government and the department of emergency management, emergency reduce losses, nearly 10 years national earthquake emergency, for example, the same magnitude 7 earthquake yushu (3000 deaths), Haiti (deaths in hundreds of thousands of people), jiuzhaigou (19 deaths), New Zealand to 0 (deaths) and ya (217 deaths) but the death toll from the earthquake landslide[16], while the death toll from the earthquake and regional population density, and geology. However, the most important thing is emergency measures taken by government after the occurrence of an earthquake emergency and the prediction of later situation development. Among them, New Zealand made the best emergency decisions, so as to minimize the casualties in earthquake. It can be seen from the results of many earthquake emergencies in China that national emergency handling and decision-making ability is improving. Thus it can be seen that it plays a vital role in the emergency decision-making.

Although this method is relatively reasonable, we still need to pay attention to one thing in the future: it can be improved in combination with prospect theory in the future, because the research method in this paper does not take into account the behavior factors of decision makers, which may produce unreasonable decision analysis results. Although the combination of calculation in this paper is relatively reasonable, it has not been verified by a large number of enterprises, so the science needs to be further investigated.

\section{Acknowledgments}

The authors acknowledge the support of the Philosophy and Social Science Foundation of Guangdong Province, China (Grant No. GD19CGL07), the Soft Science Research Project of Guangdong Province, China (Grant No. 2020A1010020034).

\section{References:}

1. M.Neocleous,Critique of security, Edinburgh
University Press, Edinburgh, Britain,2008

2. B. Meng and H.Guiming,"A Flood-control Decision Support System

3. M.Y.Li and P. P.Cao,"Extended TODIM method for multi-attribute risk decision making problems in emergency response,"Computers \& Industrial Engineering,vol.135,no.9,pp.1286-1293,2018.

4. N.Rahman and G.L.D.Feis,"Strategic decisionmaking: models and methods in the face of complexity and time pressure,"Journal of General Management,vol.35,no. 4,pp. 43-59,2009.

5. L.Yu and K. Keunglai,"A distance-based group decision-making methodology for multi-person multi-criteria emergency decision support,"Decision Support Systems,vol.51,no.2, pp.307-315,2011.

6. Y.Liu,Z.P.Fan and Y.Yuan,"Multiple attributes risk decision making method for emergency response,"Operations Research and Management ence,vol.22,no.1,pp.23-28,2013

7. K.Amailef and J. Lu,"Ontology-supported case-based reasoning approach for intelligent $\mathrm{m}$-Government emergency response services,"Decision Support Systems,vol, 55,no.1,pp.79-97,2013.

8. X.H.Xu,Z.J.Du and X.H.Chen,"Consensus model for multi-criteria large-group emergency decision making considering non-cooperative behaviors and minority opinions, Decision Support Systems,vol.79, no.1,pp.150-160,2015.

9. D.Mendonca,R.Rush and W.A.Wallace,"Timely knowledge elicitation from geographically separate, mobile experts during emergency response,"Safety Science,vol.35,no.1,pp.193-208,2000.

10. G.Fu,"A fuzzy optimization method for multicriteria decision making: An application to reservoir flood control operation.,"Expert Systems with Applications, vol.34,no.1,pp.145-149,2008.

11. J.Tian,W.Z.Ma and Y.L.Wang,"Particle Swarm Optimization for Dynamic Scheduling of Emergency Material Distribution,"Systems Engineering-Theory \& Practice,vol.31,no.5,pp.898-906, 2011.

12. A.Chakraborty," Markov Chain Monte Carlo," Resonance, vol,7,no.5,pp. 66-75,2002.

13. C.Liu, Z.Chen and Y.Gong, "Site selection of emergency material warehouse under fuzzy environment," Journal of Central South University, no.6,pp.1610-1615,2013.

14. T.C.Wang and H.D.Lee, "Developing a fuzzy TOPSIS approach based on subjective weights and objective weights," Expert Systems with Applications, vol.36,no.5,pp.8980-8985,2009.

15. Y.Liu, Z.P.Fan and Y.Zhang, "Risk decision analysis in emergency response: A method based on cumulative prospect theory," Computers \& Operations Research,vol.42,no.2,pp. 75-82,2014. 
16. S.Shapira, L.Aharonson-Daniel and Y.Bar-Dayan, "Anticipated behavioral response patterns to an earthquake: The role of personal and household characteristics, risk perception, previous experience and preparedness," International Journal of Disaster Risk Reduction,vol.31,pp.1-8,2018 